\title{
Moedas complementares digitais e políticas públicas durante a crise da COVID-19
}

Lauro Gonzalez 1

Adrian Kemmer Cernev 1

Marcelo Henrique de Araujo 1

Eduardo H. Diniz 1

1 Fundação Getulio Vargas / Escola de Administração de Empresas de São Paulo, São Paulo / SP - Brasil

Programas de renda básica têm sido utilizados em todo o mundo como uma ferramenta para mitigar os efeitos adversos da crise da COVID-19. No Brasil, a implementação de iniciativas federais de renda básica emergencial (RBE) enfrenta um duplo desafio: a logística de distribuição de dinheiro e os critérios de elegibilidade dos cidadãos. No entanto, iniciativas de moedas complementares existem há muitos anos no Brasil, estando associadas especialmente aos bancos comunitários, os quais operam no nível local e possuem conhecimento mais aprofundado sobre as necessidades dos moradores. Este artigo analisa o uso de moedas digitais complementares no enfrentamento de desafios de distribuição de renda. Apresentamos o caso da moeda complementar digital Mumbuca E-Dinheiro, adotada pelo município de Maricá (RJ). Discutimos como esta iniciativa permitiu a distribuição de renda de forma rápida e segura com o objetivo de mitigar os efeitos da pandemia da COVID-19 no Brasil. Sugerimos que, no momento atual, a RBE poderia ser paga através do E-dinheiro, começando pelos municípios nos quais ele já atua e depois se expandindo para os demais. A interoperabilidade com outros atores do ecossistema de pagamentos e articulações com governos locais são medidas adicionais para dar escala ao uso das moedas complementares digitais no combate à crise do coronavírus.

Palavras-chave: COVID-19; moeda complementar digital; distribuição de renda; Mumbuca E-Dinheiro; renda básica emergencial; políticas públicas.

\section{Monedas complementarias digitales y políticas públicas durante la crisis de COVID-19}

Los programas de ingresos básicos se han utilizado en todo el mundo como una herramienta para mitigar los efectos adversos de la crisis de COVID-19. En Brasil, la implementación de iniciativas federales de ingresos básicos de emergencia enfrenta un doble desafío: la logística de distribución de dinero y los criterios de elegibilidad de los ciudadanos. Las iniciativas monetarias complementarias han existido en Brasil durante muchos años y están asociadas especialmente con bancos comunitarios, instituciones que operan a nivel local y tienen un conocimiento más profundo sobre las necesidades de los residentes. Este artículo examina el uso de monedas complementarias digitales para enfrentar los desafíos de distribución de ingresos. Presentamos el caso de la moneda complementaria digital Mumbuca E-Dinheiro adoptada por el municipio de Maricá (RJ) y discutimos cómo esta iniciativa permitió la distribución de ingresos de manera rápida y segura para mitigar los efectos de la pandemia de COVID-19 en Brasil. Sugerimos que, en el momento actual, los ingresos básicos de emergencia podrían pagarse a través de la plataforma E-Dinheiro, comenzando por los municipios en que esta plataforma ya opera y luego expandiéndose a los demás. La interoperabilidad con otros actores del ecosistema de pagos y los vínculos con los gobiernos locales son medidas adicionales para ampliar el uso de monedas complementarias digitales en la lucha contra la crisis del coronavirus.

Palabras clave: COVID-19; moneda complementaria digital; distribución de ingresos; Mumbuca E-Dinheiro; ingreso básico de emergencia; políticas públicas. 


\section{Digital complementary currencies and public policies during the COVID-19 pandemic}

Basic income programs have been used worldwide as a tool to mitigate the adverse effects of the COVID-19 pandemic. In Brazil, the implementation of federal emergency basic income initiatives faces a twofold challenge: money distribution logistics and eligibility criteria. This paper analyses the use of digital complementary currencies (DCC) to face these challenges. Complementary currencies have long existed in Brazil as part of community banks. The latter are institutions that operate at the local level and have better information regarding residents' needs. We present the case of Mumbuca E-Dinheiro, a DCC adopted by the municipality of Maricá (RJ), and discuss how this initiative has enabled quick and safe cash distribution aimed at mitigating the effects of the COVID-19 pandemic in Brazil. We suggest that, at present, basic emergency income could be distributed through the E-Dinheiro platform, starting with the municipalities in which it already operates and then expanding to others. Interoperability with other actors in the payment ecosystem and connections with local governments are additional actions to scale up the use of digital complementary currencies to combat the coronavirus crisis.

Keywords: COVID-19; digital complementary currency; social cash transfers; Mumbuca E-Dinheiro; basic emergency income; public policies.

\section{INTRODUÇÃO}

A pandemia do novo coronavírus criou inúmeros focos de crise que rapidamente se espalharam por praticamente todas as áreas. A porção mais visível dos problemas relaciona-se à saúde e aos efeitos econômicos da crise. O denominador comum das listas de problemas é a exigência de respostas rápidas por parte do Estado. No entanto, entre a formulação de políticas públicas e sua efetiva implementação há uma distância cronológica que se mostra incompatível com a mitigação dos efeitos da pandemia (Hudson, Hunter, \& Peckham, 2019; Lotta \& Gonzalez, 2020).

Visando mitigar os efeitos da crise da COVID-19, programas de renda básica têm sido introduzidos mundialmente (Jacob \& Boyd, 2020; Tomazelli \& Fernandes, 2020). No Brasil, um dos maiores desafios destes programas concerne à logística de distribuição dos recursos, isto é, o processo de fazer o dinheiro chegar aos grupos mais pobres, justamente os mais afetados pela crise (Gonzalez \& Cernev, 2020). Perguntas sobre como liberar os recursos sem causar aglomerações ou, ainda, como selecionar os que estão fora do Cadastro Único (CadÚnico) permanecem abertas. Portanto tirar medidas do papel e fazê-las chegar à ponta, ao território ou local permanece como o maior desafio (Lotta \& Gonzalez, 2020).

Neste contexto, uma iniciativa que pode contribuir para melhorar a implementação de programas de renda básica emergencial (RBE) para mitigar os efeitos da COVID-19 são as moedas complementares (MC), também conhecidas como moedas sociais, alternativas ou comunitárias (neste artigo, todos esses termos serão tratados com o mesmo significado). Estas moedas visam atender comunidades específicas e são concebidas, emitidas e usadas por agentes locais a fim de complementar o sistema monetário (oficial) nacional (Diniz, Cernev, Daneluzzi, \& Rodrigues, 2018; Meyer \& Hudon, 2019). Tais moedas alternativas são frequentemente desenvolvidas para atender necessidades sociais para as quais as moedas nacionais não são projetadas (e.g. promoção do desenvolvimento local, comportamento sustentável, inclusão financeira, ampliação do capital social da comunidade, combate à exclusão social, entre outros) (Amato \& Fantacci, 2020; Blanc, 2011; Joachin \& Klopfert, 2014; Meyer \& Hudon, 2019; Seyfang, 2004).

Moedas complementares digitais (MCD) são aquelas que incorporam as tecnologias da informação como forma de viabilizar sua circulação no território em que operam (Alves, Diniz, Cernev, \& 
Nascimento, 2014), e possuem três elementos estruturantes: um agente emissor, um meio digital e circulação delimitada (Diniz, Siqueira, \& Van Heck, 2019).

Este é o caso da moeda Mumbuca implementada pela Prefeitura Municipal de Maricá/RJ desde 2013, a primeira moeda complementar originalmente digital do Brasil (Cernev \& Proença. 2016). Inicialmente projetada para circular com cartões magnéticos e máquinas de captura de transações por cartões (POS), a Mumbuca viabiliza a transferência direta mensal de recursos sociais da Prefeitura Municipal de Maricá a milhares de beneficiários cadastrados em seu programa de assistência, de forma rápida e imediata (Alves et al., 2014), tendo aprimorado a logística de pagamento para as pessoas de baixa renda.

Tanto a Mumbuca como dezenas de outras moedas complementares, inicialmente baseadas em papel-moeda, tornaram-se modelos híbridos de mobile money e mobile payments, com a introdução da plataforma digital e do aplicativo E-Dinheiro, desenvolvida e gerenciada pelo do Instituto Banco Palmas e da Rede Brasileira de Bancos Comunitários (Cernev, 2019; Cernev \& Diniz, 2020). Por sua origem, finalidade e eficácia, o Mumbuca E-Dinheiro evidencia sua potencial utilidade em um momento que se busca, pela implementação de um esquema de distribuição de renda básica para mitigar os efeitos da crise da COVID-19.

As próximas seções deste artigo estão estruturadas da seguinte forma: a seção 2 apresenta os principais conceitos relacionados às moedas complementares e suas características, incluindo potenciais benefícios sociais; a seção 3 expõe o caso da moeda complementar digital (MCD) Mumbuca E-Dinheiro; a seção 4 debate o potencial desta iniciativa para superar os desafios da RBE; a seção 5 discute como dar escala às iniciativas de MCD no contexto de enfrentamento dos efeitos da pandemia; a seção 6 apresenta as conclusões finais do trabalho.

\section{MOEDAS COMPLEMENTARES}

O sistema monetário e suas moedas associadas são uma criação social que evoluiu ao longo da história da humanidade e, atualmente, compõem o sistema de pagamentos e de transações comerciais mais aceito mundialmente. Neste sistema, o Estado é o ator central na emissão da moeda (Gómez \& Demmler, 2018). Embora as moedas nacionais ('oficiais') sejam amplamente aceitas e institucionalizadas, alguns autores apontam várias desvantagens no seu uso, como o aumento de disparidades econômicas e sociais, especulação e acumulação, que levam a padrões de consumo insustentáveis (Gómez \& Demmler, 2018; Meyer \& Hudon, 2019).

Neste contexto, emerge o fenômeno das moedas complementares, cujo nome deriva do fato de atuarem de maneira complementar às moedas oficiais (Alcantara \& Dick, 2017). Elas constituem uma nova forma de troca monetária, a qual desafia “[...] a ontologia do dinheiro e suas funções na sociedade, revivendo o debate em torno do papel dos sistemas monetários a da sua utilidade aos interesses comuns dos povos [...]" (Meyer \& Hudon, 2019, p. 1).

O conceito de MC pode ser definido como um sistema monetário que suplementa as moedas oficiais nacionais ou transnacionais (Lietaer, 2001). Esta forma de moeda é concebida e emitida por cidadãos, organizações não governamentais (ONGs), empresas e a própria administração pública (Ingham, 2004; Meyer \& Hudon, 2019). É essencialmente usada para negociação e troca de bens e serviços que muitas vezes não são valorizados pelo sistema de preços de mercado (Gomez \& Helmsing, 2008; Meyer \& Hudon, 2019). 
O fenômeno das moedas complementares não é novo, tendo sido observado em vários países há décadas. Atualmente existem mais de 6.000 iniciativas criadas em todo o mundo que utilizam moedas complementares (Diniz et al., 2019). Estas moedas geralmente são projetadas para abordar questões relacionadas ao combate da exclusão, ou à promoção do desenvolvimento sustentável local (Diniz et al., 2018).

Por um longo tempo, MC caracterizaram-se por um comportamento anticíclico, tendendo a serem adotadas em tempos de crise econômica, quando há desemprego e acesso restrito aos principais sistemas financeiros para a população mais vulnerável - sua taxa de adoção é reduzida quando o ciclo econômico melhora (Diniz et al., 2018; Place, Calderon, Stodder, \& Wallimann, 2018). No entanto, na última década, um crescimento constante destas moedas foi observado em todo o mundo. Na França, por exemplo, o número delas saltou, entre 2010 e 2018, de 5 para 74, juntamente com o aumento expressivo do número de usuários (Blanc \& Lakócai, 2020). O surgimento de MCD que utiliza a tecnologia blockchain - base de funcionamento de criptomoedas - sugere que as moedas complementares podem estar entrando em uma nova era de consolidação (Uzureau, Furlonger, \& Merji, 2019).

\subsection{Moedas complementares e benefícios sociais}

A articulação entre as organizações da sociedade civil e os governos locais tem demonstrado o potencial de MC como uma maneira de aliviar os problemas sociais. Kobayashi, Miyazaki, e Yoshida (2017) demonstram iniciativas de MC que contribuem para o desenvolvimento da reciclagem, da proteção do meio ambiente natural, da atividade cívica e de outros benefícios sociais.

Alguns casos ilustrativos podem demonstrar o potencial das moedas complementares como uma ferramenta para a promoção de benefícios sociais. Fureai Kippu (que significa "bilhete para um relacionamento solidário") é uma moeda complementar do Japão que fornece créditos às pessoas que ajudam os idosos da comunidade (Diprose, 2020). A Moeda Verde, que circula em Santa Cruz da Esperança/SP, fornece renda às famílias vulneráveis e educa jovens sobre os benefícios do uso correto de resíduos sólidos (França, Amato, Gonçalves, \& Almeida, 2020).

Em tempos de MCD e criptomoedas, surgem projetos que visam à recuperação econômica após a crise da COVID-19. Um exemplo vem de comunidades empobrecidas de Mukuru, no Quênia, onde pessoas que ficaram sem o dinheiro oficial do país, devido às crises epidemiológicas, conseguem obter acesso aos bens básicos usando o Sarafu, uma moeda complementar baseada em blockchain (Chibwara, 2020).

Apesar da grande independência desta modalidade de moeda em relação aos governos, a maioria dos exemplos mencionados depende de articulações com políticas públicas. Blanc e Fare (2013) afirmam que as relações de governos com moedas complementares são complexas e ambíguas. Na opinião dos autores, os governos locais podem promover o pagamento de vários serviços em moedas complementares como uma forma potencialmente poderosa de suporte indireto. Do mesmo modo, o governo do Reino Unido reconheceu parcerias entre iniciativas de $\mathrm{MC}$ e diferentes níveis de governo como uma alternativa para combater a pobreza, expandir a assistência à saúde, criar laços sociais, promover cuidados ambientais e melhorar os serviços sociais em geral (Seyfang, 2006).

ReVISTA DE AdMinistração PÚblica | Rio de Janeiro 54(4):1146-1160, jul. - ago. 2020 


\subsection{Moedas complementares no Brasil}

No contexto brasileiro, os chamados bancos comunitários de desenvolvimento têm desempenhado um papel de liderança na disseminação e expansão do modelo de MC (Diniz, Cernev, \& Nascimento, 2016). O primeiro banco comunitário no Brasil, o mais conhecido e amplamente estudado, é o Banco Palmas, fundado em 1998 no conjunto Palmeira, localizado na região periférica da cidade de Fortaleza/CE. O banco utiliza o microcrédito e a MC Palma como elemento fundamental de seu funcionamento (Diniz et al., 2016).

Apesar das conquistas do Banco Palmas com a utilização da MC Palma, Diniz et al. (2016), em análise de dados coletados com gerentes de bancos comunitários de desenvolvimento, identificam uma série de dificuldades e problemas no uso diário de moedas complementares em papel. Desse modo, a tecnologia da informação passou a ser considerada uma alternativa para a melhoria de MC em papel, visando torná-la digital com a adoção de tecnologias emergentes (Diniz et al., 2016).

O Banco Palmas liderou, assim, a iniciativa para a criação da Rede Brasileira de Bancos Comunitários (REDE), atualmente composta por 103 bancos que seguem o marco teórico conceitual dos bancos comunitários de desenvolvimento (Instituto Banco Palmas, 2020). Juntamente com a REDE, o Banco Palmas também fundou o Instituto E-Dinheiro Brasil, em novembro de 2014 e uma plataforma digital que utiliza tecnologia digital móvel para moedas complementares, fornecendo uma conta corrente digital para os usuários de MC e oferecendo ainda uma variedade de serviços.

Mais do que a mera digitalização de MC nas localidades, o E-Dinheiro é visto por líderes dos bancos comunitários de desenvolvimento $(\mathrm{BCD})$ como uma tecnologia social que promove a economia solidária, uma vez que ele permite a criação de novos BCDs e moedas sociais, baseado no uso de um simples microcomputador local (Cernev \& Diniz, 2020). Conceitualmente o E-Dinheiro é um modelo híbrido de mobile money e mobile payments que amplia o efeito potencial das moedas complementares físicas, tanto em alcance quanto em uso efetivo (Cernev \& Diniz, 2020), sendo atualmente adotado por 48 bancos comunitários de desenvolvimento, incluindo o Banco Mumbuca, cuja MCD Mumbuca será apresentada a seguir.

\section{MUMBUCA E-DINHEIRO}

A Mumbuca foi lançada em dezembro de 2013, seguindo uma regulamentação municipal aprovada pela Câmara Municipal de Maricá, no Estado do Rio de Janeiro, Brasil (Faria, 2018). O Banco Comunitário Popular de Maricá (Banco Mumbuca) foi contratado para gerenciar a operação da MCD, entregando cartões magnéticos aos beneficiários e atraindo comerciantes locais para aceitarem o cartão Mumbuca usando máquinas POS (Alves et al., 2014).

Por se situar em uma localidade que se insere na região de exploração de petróleo, o município de Maricá começou a receber royalties. De modo a fomentar o desenvolvimento econômico da região e beneficiar famílias de baixa renda, a Prefeitura Municipal de Maricá decidiu criar um banco comunitário no município, contratando, para tanto, o Instituto Banco Palmas.

A ideia principal relacionada ao banco comunitário e à sua moeda complementar era incentivar a circulação dos recursos oriundos dos royalties dentro do próprio município, impulsionando a economia e o desenvolvimento da região (Cernev \& Proença, 2016; Cernev, 2019) por meio da alocação de parte dos recursos financeiros dos royalties de petróleo para famílias de baixa renda do município. Com este fim, a Prefeitura informa ao Banco Mumbuca quem são os beneficiários ativos em seus programas 
de assistência, de acordo com sua situação socioeconômica, e transfere os recursos financeiros para o banco, que, por sua vez, distribui-os às famílias cadastradas no programa.

Após a abertura de cadastro no Banco Mumbuca, o beneficiário recebe uma conta digital em seu nome e um cartão magnético. Este cartão pode ser utilizado para pagamento em diversos estabelecimentos comerciais cadastrados no programa. Todo o processo beneficia a comunidade com acréscimos de renda e impulsiona as vendas do comércio local, que ganha competitividade em relação a outros estabelecimentos maiores (Alves et al., 2014; Cernev, 2019).

Após seis meses de operação, o Banco Mumbuca havia cadastrado 104 estabelecimentos comerciais do município (mercados, farmácias, distribuidores de água e gás, lojas de materiais de construção, entre outros), e seu programa de assistência municipal possuía 7.576 beneficiários, que recebiam $\mathrm{R} \$ 70,00$ por mês para utilizar na rede de estabelecimentos credenciados, valor que aumentou para R\$ 130,00 em julho de 2017 (Cernev, 2019).

No primeiro semestre de 2018, a Prefeitura do Município de Maricá decidiu aprimorar o projeto Mumbuca usando a plataforma E-Dinheiro, já adotada por outros bancos comunitários. Com a mudança, o Banco Mumbuca passou a gerenciar o projeto por meio de uma plataforma digital, "[...] desde a emissão da moeda social até a relação contratual com os agentes envolvidos [...]" (Cernev, 2019, p. 4).

Com essa mudança, os usuários começaram a utilizar o aplicativo E-Dinheiro para variadas transações financeiras, desde pagamentos nos estabelecimentos locais até transferências diretas entre pessoas. Em virtude de potenciais restrições ao uso do aplicativo, manteve-se a emissão complementar gratuita de novos cartões (em substituição aos anteriores), sempre vinculados a uma conta de usuário na plataforma E-Dinheiro. O novo cartão Mumbuca possui tecnologia near field communication (NFC) ou contactless (sem contato), que permite a realização de pagamentos sem contato físico, uma vez que são feitos no smartphone do estabelecimento comercial cadastrado, dispensando o uso das máquinas POS para transações (Cernev, 2019). Além das melhorias do ponto de vista sanitário, as novas tecnologias propiciaram também a realização de transações mais seguras.

Com a adoção do E-Dinheiro, qualquer morador de Maricá pode abrir uma conta digital pré-paga e usar o Mumbuca como forma de pagamento por meio do aplicativo. Em 2018, foram quase 23.000 contas abertas (Banco Mumbuca, 2019). Além disso, o número de transações aumentou cinquenta vezes, incentivando mais comerciantes locais a ingressarem na plataforma E-Dinheiro e envolvendo mais de 1.300 estabelecimentos comerciais registrados (Cernev, 2019).

\section{RENDA BÁSICA EMERGENCIAL E 0 CASO DO MUMBUCA E-DINHEIRO}

Com o advento da pandemia da COVID-19, um conjunto de medidas econômicas foram criadas visando, especificamente, minimizar os impactos financeiros na população mais afetada, especialmente a de baixa renda, incluindo desempregados, pequenos comerciantes, trabalhadores informais e outras pessoas que se encontram em situação de vulnerabilidade. Os esquemas de RBE, direcionando recursos financeiros para os cidadãos, foram adotados em todo o mundo (Jacob \& Boyd, 2020; Tomazelli \& Fernandes, 2020).

No Brasil, a RBE de R 600 por mês, anunciada pelo Governo Federal e aprovado pelo Congresso, deve ser paga pela Caixa Econômica Federal (Caixa) por três meses. As estimativas iniciais do governo giravam em torno de 30 milhões de pessoas elegíveis; entretanto, as últimas notícias disponíveis dão 
conta de que mais de 50 milhões de pessoas já receberam a primeira parcela do benefício, o que representa cerca de $50 \%$ da população ocupada do país.

A implementação envolve duas etapas que precisam ser executas adequadamente: cadastro/análise de elegibilidade e logística de pagamento (Gonzalez \& Cernev, 2020). Ou seja, primeiramente, é preciso cadastrar e analisar, ainda que minimamente, a elegibilidade ao recurso, ou seja, o cumprimento dos critérios pré-definidos para se beneficiar da assistência. Por exemplo, existem limites de renda familiar que devem ser considerados na análise da elegibilidade dos beneficiários: uma renda familiar mensal per capita inferior a meio salário-mínimo, ou inferior a três salários-mínimos no total, além de uma renda tributável máxima de $\mathrm{R} \$ 28.559,70$ em 2018. O reconhecimento dos potenciais beneficiários é um grande desafio, considerando que apenas parte deles está atualmente registrada no Cadastro Único do Governo Federal (CadÚnico). Muitas pessoas que não fazem parte do CadÚnico, o qual tem como unidade a 'família' - conceito que pode ser fluído (Ribeiro, 2017) - são elegíveis para o benefício emergencial. Para superar este desafio, a Caixa desenvolveu e lançou seguidamente dois aplicativos para dispositivos móveis: primeiramente o Caixa Auxílio Emergencial, para que as pessoas pudessem reivindicar a assistência por autodeclaração. Poucos dias depois, lançou o segundo aplicativo móvel, o Caixa Tem, destinado a facilitar consultas e transações envolvendo os recursos de emergência.

A segunda etapa do processo é a transferência efetiva dos recursos ou a logística de pagamento. Trata-se de outro desafio, pois, além da incompletude do CadÚnico discutida acima, nem todos os potenciais beneficiários possuem ou utilizam contas bancárias (Demirgüç-Kunt, Klapper, Singer, Ansar, \& Hess, 2018). Segundo Bader e Savoya (2013) “[...] o novo paradigma logístico do sistema financeiro demanda a necessidade de aproximar-se do cliente final [...]” (p. 210), e a “[...] aproximação pode ser por meio de parcerias com atores locais [...]" (Gonzalez, Diniz, \& Pozzebon, 2015). Nota-se que existe uma diferença entre o acesso aos serviços financeiros em geral, e às contas bancárias em particular, e seu efetivo uso (Aker, Boumnijel, McClelland, \& Tierney, 2016). No caso da RBE, a opção foi promover uma rápida bancarização, ou seja, abertura de novas contas bancárias exclusivamente pela Caixa.

Apesar dos esforços da Caixa, vários problemas surgiram. Primeiramente, a demora no recebimento dos recursos. Segundo, mesmo após a chegada, a estratégia de pagamento adotada acabou gerando longas filas e aglomerações de pessoas em agências bancárias e órgãos governamentais, este último para regularização dos CPFs; tudo isso durante um período crítico no qual as autoridades de saúde recomendam o distanciamento social. Por fim, a elegibilidade vem sendo contestada, pois algumas pessoas recebem indevidamente, enquanto outras, que incontestavelmente têm direito, não são alcançadas pelo auxílio financeiro (Torrente, 2020).

Diante dos problemas relatados pela aplicação do esquema de RBE adotado pelo governo, a experiência do Banco Mumbuca evidencia o valor das MCDs no combate aos efeitos negativos da COVID-19. O pacote emergencial implementado no município de Maricá incluiu um conjunto amplo de medidas: ampliação temporária do benefício assistencial Mumbuca para $\mathrm{R} \$ 300$ durante três meses; antecipação do abono natalino para 40 mil beneficiários (Prefeitura Municipal de Maricá, 2020a).

Em Maricá, todos os pagamentos foram feitos usando a MCD Mumbuca, que tem paridade de um para um com o Real. O uso da Mumbuca ganha especial relevância durante a crise da COVID-19, na medida em que a transferência de recursos e a realização de pagamentos entre pessoas e entidades comerciais locais podem ser feitas remotamente, ou pelo menos sem contato físico, via aplicativo móvel ou cartão NFC. 
Diferentemente da iniciativa do Governo Federal, os pagamentos realizados via MCD no município de Maricá chegaram aos beneficiários em tempo hábil e sem a necessidade de aglomeração humana nem de riscos adicionais para a saúde pública, tanto para o recebimento dos recursos, quanto para a sua utilização. A razão primordial do sucesso da operação pode ser creditada às condições descritas anteriormente: conhecimento prévio da elegibilidade dos beneficiários (cidadãos e pequenos empresários) e a disponibilidade de um instrumento de pagamento eletrônico ao alcance dos cidadãos - resultado da existência e da circulação da Mumbuca E-Dinheiro.

Vale mencionar que o Banco Mumbuca oferece ainda microcrédito em MCD, o que amplia a sua utilização e atinge, inclusive, pessoas não beneficiárias dos programas municipais (Cernev, 2019). Este aspecto relaciona-se à dimensão de elegibilidade, pois envolve o bom conhecimento da economia local (Meyer \& Hudon, 2019), incluindo bases de dados atualizados que permitem identificar os agentes econômicos mais fragilizados neste momento de crise. De fato, o Banco Mumbuca, com experiência em programas voltados para famílias (bolsa Mumbuca) e para indivíduos (bolsas dos Programas Gestante, Jovem Solidário, e Renda Básica de Cidadania) (Prefeitura Municipal de Maricá, 2020b), demonstra capacidade potencial para diversificar os benefícios prestados e para atender políticas específicas de ajuda econômica. Desse modo, ao ir além dos sistemas de pagamento e incluir outros serviços, a Mumbuca promove a inclusão financeira, entendida como o acesso e o uso de produtos e serviços financeiros pela população de baixa renda, geralmente excluídos do sistema financeiro tradicional, de forma a contribuir para sua qualidade de vida (Mazumder \& Lu, 2015; Roa, 2015; Sela, Gonzalez, \& Christopoulos, 2020).

Destaca-se que a competência em lidar com a elegibilidade decorre da proximidade com o público alvo (Gonzalez et al., 2015), característica intrínseca à tecnologia social dos bancos comunitários e fortalecida pelo uso da MCD, conforme ressaltado anteriormente na fala de Joaquim Mello (Cernev \& Diniz, 2020). Ademais, o sistema de pagamento digital das MCDs contribui para o bom funcionamento da logística de pagamento (Bader \& Savoya, 2013), e o mecanismo de atuação territorial das MCDs contribui para o desenvolvimento local. Em suma, a MCD, digital por definição, está diretamente relacionada à logística, embora também contribua para o cadastro e para a elegibilidade.

A atual pandemia e seus efeitos representam um forte choque adverso, talvez o maior em gerações, para todos os países. As magnitudes de tais choques distribuem-se assimetricamente e afetam significativamente a população de baixa renda, o que ocorre, justamente, porque esta população é a menos protegida pelos mecanismos tradicionais de mercado, tais como o uso de poupança acumulada ou empréstimos emergenciais (Morduch, 1999). O caso da Mumbuca E-Dinheiro evidencia como a moeda complementar digital, parte integrante da tecnologia social dos BCDs, pode expandir a inclusão financeira, de forma a contribuir para a mitigação dos efeitos negativos desta crise nas pessoas mais pobres, colaborando tanto na dimensão da elegibilidade como na execução (logística) do pagamento. Se a experiência de Maricá for replicada para outros municípios, é possível que haja maior êxito em fazer a RBE chegar a quem precisa.

\section{MEDIDAS PARA GERAR ESCALA}

Dada a dimensão da crise enfrentada, as ações do governo precisam ter a escala necessária para mitigar os efeitos negativos da pandemia, sobretudo para a população de menor renda. Na sequência, serão apresentadas medidas concretas que propiciariam escala às soluções envolvendo as MCDs e a 
RBE, iniciando por aquela com maior facilidade de execução: a articulação de soluções com histórico operacional de sucesso e que alcançam o público alvo desejado, em especial com a plataforma E-Dinheiro. Vale dizer que as medidas consideram as duas dimensões anteriormente destacadas: cadastro/elegibilidade e execução/logística de pagamentos.

\subsection{Articulação com o ecossistema de tecnologia e pagamentos}

A tecnologia é uma maneira eficaz de escalar serviços em rede (Diniz \& Cernev, 2019). Uma forma de ampliar o alcance das soluções públicas de combate aos efeitos da pandemia é a adoção de diferentes tecnologias, apoiadas por diferentes instituições e arranjos de pagamento. Contudo, tanto a construção de novos serviços - como os novos aplicativos da Caixa -, quanto o processo de adoção e efetiva utilização pelos usuários podem encontrar restrições, demandando considerável tempo e esforço adicional.

Assim sendo, o apropriado no momento é articular soluções existentes, ou seja, já operacionais e que alcançam o público alvo desejado. A plataforma tecnológica E-Dinheiro, anteriormente descrita, já atende milhares de pessoas de baixa renda em mais de 40 municípios do país, sendo imediatamente escalável para atender mais usuários, uma vez que o sistema opera em nuvem (cloud computing) com uso de redes de dados e aplicativos móveis. Na computação em nuvem, o acréscimo ou redução de capacidades - como processamento e armazenamento - pode ser executado por meio de poucos cliques em uma página de controle (dashboard) via web, com ativação instantânea. Portanto a primeira medida seria efetivar o pagamento da RBE através do E-dinheiro nos municípios nos quais ele já atua, contribuindo, assim, para o aprimoramento da logística de pagamentos em tais localidades.

A segunda medida seria replicar o E-Dinheiro para mais localidades, algo tecnologicamente viável, na medida em que, para operar tal serviço, basta um microcomputador habilitado na localidade de implantação (Cernev \& Diniz, 2020). Com o apoio da metodologia já existente dos BCDs, associada à tecnologia do E-Dinheiro, os processos de replicação seriam agilizados.

Além disso, a ideia de uma solução para todo o país é exatamente usar a pluralidade de instituições locais existentes (Gonzalez \& Cernev, 2020), incluindo parceiros locais que poderiam associar-se à iniciativa, tais como agentes de crédito ligados às instituições de microfinanças de várias partes do país, que já possuem contato, experiência e interação com a população de menor renda (Gonzalez et al., 2015). Para agilizar o processo, poderiam se formar parcerias entre a rede de BCD e a Associação Brasileira de Entidades Operadoras de Microcrédito e Microfinanças (ABCRED), que já manifestou interesse em colaborar na implementação da RBE (Bolzani, 2020).

Vale ressaltar que as medidas acima descentralizariam o modus operandi da RBE, tanto na elaboração do cadastro/elegibilidade, quanto na execução do pagamento. Em vez de se aglutinarem em agências da Caixa, os beneficiários poderiam contar também com as equipes dos BCDs e com os agentes de crédito.

A terceira medida seria tornar a plataforma E-Dinheiro interoperável com outras fintechs já consolidadas no mercado. Do ponto de vista tecnológico, a integração seria relativamente simples e rápida, podendo ser incluídos, por exemplo, os serviços bancários oferecidos pela Caixa. Assim, a $\mathrm{RBE}$ recebida na plataforma E-Dinheiro poderia ser transferida para as contas de usuários e estabelecimentos em outros bancos ou instituições de pagamento. Isso permitiria o pagamento de contas, compras e transferências. Ou seja, as contas digitais seriam utilizadas para aprimorar a 
dimensão da logística de pagamento dos recursos emergenciais e seu posterior uso pelo cidadão, de forma complementar e integrada à atual solução governamental.

A interoperabilidade do E-Dinheiro com outros serviços de pagamento e bancos proporcionaria uma significativa redução no tempo e no esforço de alcançar o público alvo pretendido, uma vez que estas pessoas já possuem acesso a algum serviço de pagamento, não necessariamente bancário (Gonzalez \& Cernev, 2020). De certa forma, esta proposta seria similar ao funcionamento do sistema de pagamentos instantâneos, denominado PIX, capitaneado pelo Banco Central do Brasil (BACEN), cujo início de operação está previsto para o final de 2020.

Seria oportuno, no presente momento, acelerar a implementação do PIX. Nesse ecossistema de transferências digitais funcionando ininterruptamente, a interoperabilidade entre bancos e fintechs com mais de 500 mil clientes é uma característica central. Ou seja, seria mais rápido e fácil o envio de recursos via contas digitais ou bancárias. Os beneficiários, por sua vez, também poderiam efetuar pagamentos remotos ou transferências diretas entre pessoas (peer to peer [P2P]), diminuindo a necessidade de transacionar com papel-moeda ou cartões e, por conseguinte, expor-se ao risco de transmissão do vírus. O desafio seria acelerar o desenvolvimento tecnológico dos agentes envolvidos, fintechs e bancos, algo que faz parte do cotidiano destas organizações.

Para que a articulação com o ecossistema de tecnologia e pagamentos seja efetiva, estes agentes precisariam integrar a estratégia do Governo Federal de combate aos efeitos da pandemia, complementando-a em diversas frentes, de preferência com a articulação dos governos locais. Diversas instituições e arranjos de pagamento já se prontificaram em apoiar, algumas de forma gratuita, as iniciativas emergenciais (UOL Debate, 2020), inclusive os gestores da Rede e E-Dinheiro.

\subsection{Articulação com governos locais}

Uma segunda frente de atuação visando à escala da RBE por meio de iniciativas de MCD fundamenta-se no estabelecimento de articulações com governos locais. O relacionamento com diferentes governos subnacionais pode estabelecer-se por três grandes vertentes: (i) apoio/suporte técnico às iniciativas de moedas complementares digitais; (ii) integração das moedas digitais com serviços públicos; e (iii) estabelecimento de regulação (framework legal) em nível subnacional (Blanc \& Fare, 2013).

Na primeira vertente, o apoio e suporte mencionados acima poderiam se concretizar, por exemplo, mediante acordos estabelecendo pagamento (total ou parcial) de funcionários e pagamento de despesas gerais em MCD. Governos locais poderiam ainda oferecer instalações e equipamentos para disseminar informação e a criação de redes, entre outros (Blanc \& Fare, 2013).

A segunda vertente de relacionamento constitui a integração das moedas complementares em políticas públicas e se baseia no reconhecimento do potencial destes esquemas como instrumentos para atingir determinados objetivos do governo local. Tais objetivos podem ser categorizados como sociais, ambientais e econômicos. Pensando concretamente no caso da RBE emergencial no Brasil, o E-dinheiro e outras moedas complementares podem ser utilizados para que governos locais distribuam a ajuda financeira para um público específico (pessoas em situação de vulnerabilidade, famílias de baixa renda ou outro público alvo) ou forneçam créditos para pagamentos de produtos ou serviços específicos, como impostos e produtos locais (Blanc \& Fare, 2013). Barmes e Boait (2020) afirmam que uma característica fundamental para o sucesso de moedas complementares está na aceitação dos governos locais, pelo menos em parte, para pagamentos de impostos. Esta lógica pode contribuir para

ReVIStA de Administração PÚblica | Rio de Janeiro 54(4):1146-1160, jul. - ago. 2020 
dar maior escala às MCDs. Além disso, a aproximação com o $\mathrm{BCD}$ pode render outros benefícios indiretos, como o incentivo ao comércio local, se houver conversão do benefício de moeda fiduciária em moeda local, exatamente o que acontece no caso da Mumbuca E-Dinheiro.

A terceira vertente envolve o estabelecimento de um framework regulatório para estas moedas, reconhecendo legalmente seu papel por meio de estatutos, leis, decretos ou mesmo um tratamento especial em termos de impostos (Blanc \& Fare, 2013). No Brasil, apesar de o BACEN ter produzido a nota jurídica PGBC-5927/2011 (Banco Central do Brasil [BACEN], 2011) configurando a legalidade das moedas sociais, ainda não há legislação ou regulamentação específica. O projeto de lei complementar 93/2007, para criação do Segmento Nacional de Finanças Populares e Solidárias, amplia as possibilidades de desenvolvimento dos bancos comunitários, incluindo a competência para a emissão de moeda social, no entanto, está há mais de doze anos em tramitação na Câmara dos Deputados. A falta de fundamento legal das moedas sociais e dos bancos comunitários que as emitem compromete o sistema, deixando-o em uma região cinzenta, sem segurança jurídica ou reconhecimento, desestimulando, assim, sua adoção por governos locais (Blanc \& Fare, 2013).

Justamente por isso destaca-se o caso da Mumbuca E-dinheiro pois, diferentemente de outras moedas complementares ligadas à REDE, possui uma regulação específica definida pela prefeitura de Maricá (Programa Municipal de Economia Solidária), que legaliza e institucionaliza o uso da MCD como instrumento efetivo de política pública municipal (Município de Maricá, 2013).

\section{CONCLUSÃO}

A atual pandemia da COVID-19 apresenta imensos desafios em termos de saúde e de economia para governos de todos os níveis. Dada a magnitude da crise, várias políticas públicas precisarão ser implementadas para aliviar seus efeitos negativos. A ideia de uma solução para todo o país passa exatamente por usar a pluralidade de instituições locais existentes, dentre as quais, os bancos comunitários de desenvolvimento, mas também as cooperativas, as instituições de microfinanças, fintechs e outras organizações de atuação em âmbito local. Além disso, a tecnologia será fundamental para alavancar os efeitos destas políticas durante e após a crise.

As políticas de renda básica durante a pandemia enfrentam o desafio de efetuar transferências de dinheiro de maneira ágil e eficaz para quem mais precisa. Neste caso, as moedas complementares digitais apresentam um enorme potencial em facilitar a logística de pagamentos. O caso da Mumbuca E-Dinheiro demonstra como o uso dos recursos digitais, juntamente com a infraestrutura e tecnologia social dos bancos comunitários, pode ajudar a enfrentar alguns destes desafios, melhorando a identificação e a análise dos beneficiários elegíveis e agilizando a transferência de recursos de maneira segura.

Algumas medidas concretas para dar escala aos mecanismos da MCD envolveriam a efetivação do pagamento da RBE por meio do E-dinheiro nos municípios nos quais este último já atua. Outras medidas seriam a replicação do E-Dinheiro a outros municípios e a interoperabilidade com outros atores do ecossistema de pagamentos, além da articulação com os governos locais. Logo, a expansão do uso da MCD poderia contribuir no combate à crise decorrente da pandemia da COVID-19, cujos efeitos na economia devem ir muito além do período retratado pela curva epidemiológica. 


\section{REFERÊNCIAS}

Aker, J., Boumnijel, R., McClelland, A., \& Tierney, N. (2016). Payment mechanisms and antipoverty programs: evidence from a mobile money cash transfer experiment in Niger. Economic Development and Cultural Change, 65(1), 1-37.

Alcantara, C., \& Dick, C. (2017). Decolonization in a digital age: cryptocurrencies and indigenous selfdetermination in Canada. Canadian Journal of Law and Society, 31(1), 19-35.

Alves, M., Diniz, E., Cernev, A., \& Nascimento, E. (2014, julho). Digital social money implementation by grassroots organizations: combining bottom-up and top-down strategies for social innovations. In Proceedings do $30^{\circ}$ EGOS Colloquium, Rotterdam, The Netherlands.

Amato, M., \& Fantacci, L. (2020). Complementary currencies. In S. Battilossi, Y. Cassis, \& K. Yago (Eds.), Handbook of the History of Money and Currency (pp. 501-522). Singapore: Springer Nature.

Bader, M., \& Savoia, J. (2013). Logística da distribuição bancária: tendências, oportunidades e fatores para inclusão financeira. Revista de Administração de Empresas, 53(2), 208-215.

Banco Mumbuca. (2019). Transparência - números 2018. Recuperado de https:// institutoedinheiromarica.org/numeros-2018

Banco Central do Brasil. (2011). Nota-Jurídica PGBC-5927/2011. Consultoria Penal. Procuradoria da República no Estado do Ceará. Requisição de informações. Legalidade da emissão de moeda social pelo Banco Palmas. Esclarecimentos prestados pela unidade técnica. Análise dos aspectos criminais. Conduta que se traduz em indiferente penal. Minuta de Ofício.

Barmes, D., \& Boait, F. (2020). The tragedy of growth: to protect wellbeing and avoid ecological disaster we must abandon GDP growth and transform our economic system. London: Positive Money. Recuperado de http://positivemoney.org/wpcontent/uploads/2020/05/Positive-Money-TheTragedy-of-Growth.pdf

Blanc, J. (2011). Classifying 'CCs': Community, complementary and local currencies. International Journal of Community Currency Research, 15(D),
4-10. Recuperado de http://dx.doi.org/10.15133/j. ijccr.2011.013

Blanc, J., \& Fare, M. (2013). Understanding the role of governments and administrations in the implementation of community and complementary currencies. Annals of Public and Cooperative Economics, 84(1), 63-81.

Blanc, J., \& Lakócai, C. (2020). Toward spatial analyses of local currencies: the case of France. International Journal of Community Currency Research, 24(1), 11-29.

Bolzani, I. (2020, 7 de abril), Aplicativo para auxílio emergencial pode não alcançar todos os trabalhadores, diz especialista. Folha de São Paulo, São Paulo. Recuperado de https://www1.folha. uol.com.br/mercado/2020/04/aplicativo-paraauxilio-emergencial-pode-nao-alcancar-todos-ostrabalhadores-diz-especialista.shtml

Cernev, A. (2019). Mumbuca É Dinheiro. Revista Brasileira de Casos - Gvcasos, 9(2), doc.10.

Cernev, A., \& Diniz, E. (2020). Palmas para o E-Dinheiro! A evolução digital de uma moeda social local. Revista De Administração Contemporânea, 24(5), 487-506. Recuperado de https://doi.org/10.1590/1982-7849rac2020190390.

Cernev, A., \& Proença, B. (2016). Mumbuca: a primeira moeda social digital do Brasil. Revista Brasileira de Casos - Gvcasos, 6(2), doc.15.

Chibwara, W. (2020, 25 de março). Rural villages coping with COVID-19. Grassroots Economics Blog. Recuperado de https://www.grassrootseconomics. org/post/rural-villages-coping-with-covid-19

Demirgüç-Kunt, A., Klapper, L., Singer, D., Ansar, S., \& Hess, J. (2018). The Global Findex Database 2017: measuring financial inclusion and the fintech revolution. Washington, DC: World Bank. Recuperado de https://doi.org/10.1596/978-1-46481259-0

Diniz, E., \& Cernev, A. (2019). Fintech: a sexta onda de inovações no sistema financeiro (Cap. 10). In Tigre, P. B., Pinheiro, A. M. (Org.), Inovação em Serviços na Economia do Compartilhamento. São Paulo, SP: Editora Saraiva.

Diniz, E., Cernev, A., Daneluzzi, F., \& Rodrigues, D. (2018). Social cryptocurrencies: social finance 
organizations at the new era of digital community currencies. EGOS 2018 Colloquium, 34, Tallinn, Estonia.

Diniz, E., Cernev, A., \& Nascimento, E. (2016). Mobile social money: an exploratory study of the views of managers of community banks. Revista de Administração, 51(3), 299-309.

Diniz, E., Siqueira, E., \& Van Heck, E. (2019). Taxonomy of digital community currency platforms. Information Technology for Development, 25(1), 69-91.

Diprose, G. (2020). Transacting services through time banking: renegotiating equality and reshaping work. In J. K. Gibson-Graham, \& K. Dombroski (Eds.), The Handbook of Diverse Economies (Chapter. 26, pp. 238-245). Cheltenham, UK: Edward Elgar Publishing.

Faria, L. (2018). Digitalizações de moedas sociais no Brasil e suas (pré)histórias: tensões e mediações com Estados, mercados e tecnologias. (Tese de Doutorado). Universidade Federal do Rio de Janeiro, Rio de Janeiro, RJ, Brasil.

França, A., Amato, J., Neto, Gonçalves, R., \& Almeida, C. (2020, March). Proposing the use of blockchain to improve the solid waste management in small municipalities. Journal of Cleaner Production, 244(20), 118-529.

Gómez, G. L., \& Demmler, M. (2018). Social currencies and cryptocurrencies: characteristics, risks and comparative analysis. CIRIEC-España, Revista de Economía Pública, Social y Cooperativa, 93, 265-291.

Gomez, G., \& Helmsing, A. (2008). Selective spatial closure and local economic development: what do we learn from the Argentine local currency systems? World Development, 36(11), 2489-2511

Gonzalez, L., \& Cernev, A. (2020, 2 de abril). Como fazer o dinheiro chegar a quem mais precisa? Valor Econômico. Recuperado de https://eaesp.fgv.br/ noticias/como-fazer-dinheiro-chegar-quem-maisprecisa

Gonzalez, L., Diniz, E. H., \& Pozzebon, M. (2015). The value of proximity finance: how the traditional banking system can contribute to microfinance. Innovations: Technology, Governance, Globalization, 10(1-2), 125-37.
Hudson, B., Hunter, D., \& Peckham, S. (2019). Policy Failure and the policy-implementation gap: can policy support programs help? Policy Design and Practice, 2(1), 1-14.

Ingham, G. (2004). The nature of money. Cambridge, UK: Polity Press.

Instituto Banco Palmas (2020). Rede Brasileira de Bancos Comunitários. Recuperado de https:// www.institutobancopalmas.org/rede-brasileira-debancos-comunitarios/

Jacob, A., \& Boyd, R. (2020). Addressing economic vulnerability among low-income families in America: is the basic income approach a viable policy option? Journal of Children and Poverty, 26(1), 85-99.

Joachin, H., \& Klopfert, F. (2014). Smarter than metering? Coupling smart meters and complementary currencies to reinforce the motivation of households for energy savings. Ecological Economics, 105, 89-96.

Kobayashi, S., Yoshihisa, M., \& Yoshida, M. (2017, maio). Historical transition of community currencies in Japan (Paper). In 4th International Conference on Social and Complementary Currencies, Barcelona, Spain.

Lietaer, B. (2001). The future of money: creating new wealth, work and a wiser world. London, UK: Random House Business.

Lotta, G., \& Gonzalez, L. (2020, 8 de abril). Sem implementação não há solução. Estadão (Gestão, Política e Sociedade). Recuperado de https://politica. estadao.com.br/blogs/gestao-politica-e-sociedade/ sem-implementacao-nao-ha-solucao/

Mazumder, M., \& Lu, W. (2015). What impact does microfinance have on rural livelihood? A comparison of governmental and non-governmental microfinance programs in Bangladesh. World Development, 68, 336-354

Meyer, C., \& Hudon, M. (2019). Money and the commons: an investigation of complementary currencies and their ethical implications. Journal of Business Ethics, 160, 277-292.

Morduch, J. (1999). The microfinance promise. Journal of Economic Literature, 37(4), 1569-1614.

Município de Maricá (2013). Lei $n^{\circ}$ 2.448, de 26 de junho de 2013. Institui o Programa Municipal 
de Economia Solidária, Combate à Pobreza e Desenvolvimento Econômico e Social de Maricá. Recuperado de http://www.institutobancopalmas. org/wp-content/uploads/lei-moeda-socialmumbuca.pdf

Place, C., Calderon, A., Stodder, J., \& Wallimann, I. (2018, Summer). Swiss Currency Systems: Atlas, Compendium and Chronicle of legal aspects. International Journal of Community Currency Research, 22, 85-104.

Prefeitura Municipal de Maricá. (2020a). Maricá terá pacote social e econômico contra efeitos do coronavírus de $R \$ 80$ milhões. Recuperado de https://www. marica.rj.gov.br/2020/03/21/marica-tera-pacotesocial-e-economico-contra-efeitos-do-coronavirusde-r-80-milhoes/

Prefeitura Municipal de Maricá. (2020b). Secretaria de Economia Solidária. Recuperado de https://www. marica.rj.gov.br/category/estrutura/secretarias/ economia-solidaria/

Ribeiro, M. M. (2017). Relações intergovernamentais e uso de sistemas de informação em políticas sociais no Brasil. (Tese de Doutorado). Escola de Administração de Empresas de São Paulo, Fundação Getulio Vargas, São Paulo, SP, Brasil.

Roa, M. (2015). Financial inclusion in Latin America and the Caribbean: access, usage and quality. Mexico, DF: CEMLA.

Sela, V., Gonzalez, L., \& Christopoulos, T. (2020). Construção de uma agenda de inclusão financeira à luz da Teoria Ator-Rede. Revista de Administração Pública, 54(1), 162-180.

Seyfang, G. (2006). Harnessing the potential of the social economy? Time banks and UK public policy. International Journal of Sociology and Social Policy, 26(9/10), 430-443.

Seyfang, G. (2004). Time banks: rewarding community self-help in the inner city? Community Development Journal, 39(1), 62-71.

Tomazelli, I., \& Fernandes, A. (2020, 23 de março). Ao menos 45 países já adotaram medidas para conter impactos da Covid-19 em emprego e renda. $O$ Estado de S. Paulo. Recuperado de https://economia. estadao.com.br/noticias/geral,ao-menos-45-paisesja-adotaram-medidas-para-conter-impactos-dacovid-19-em-emprego-e-renda,70003244688

Torrente, A. (2020). Ele ganhou os $\mathrm{R} \$ 600$, mas não precisa; ela precisa, mas não tem direito. UOL Notícias. Recuperado de https://economia. uol.com.br/noticias/redacao/2020/04/18/auxilioemergencial-r-600-quem-tem-direito-quemprecisa-distorcoes.htm?cmpid=copiaecola

UOL Debate (2020). Fintechs entregariam dinheiro às pessoas em dias, não semanas. Recuperado de https:// economia.uol.com.br/noticias/redacao/2020/04/27/ associacao-defende-fintechs-atuando-nadistribuicao-do-auxilio-emergencial.htm

Uzureau, C., Furlonger D., \& Merji, A. (2019, 14 de junho). Expand your token universe to create new business models. Stamford, Connecticut: Gartner Research. 


\section{Lauro Gonzalez}

ID

https://orcid.org/0000-0001-9316-6525

Doutor em Economia de Empresas; Professor na Escola de Administração de Empresas São Paulo da Fundação Getulio Vargas (FGV EAESP); Coordenador do Centro de Estudos em Microfinanças e Inclusão Financeira da Fundação Getulio Vargas (GVCemif). E-mail: lauro.gonzalez@fgv.br

\section{Adrian Kemmer Cernev}

https://orcid.org/0000-0002-6300-3976

Doutor em Administração; Professor na Escola de Administração de Empresas São Paulo da Fundação Getulio Vargas (FGV EAESP); Professor do Departamento de Tecnologia e Ciência de Dados (TDS); Pesquisador do Centro de Estudos em Microfinanças e Inclusão Financeira da Fundação Getulio Vargas (GVCemif).

E-mail: adrian.cernev@fgv.br

\section{Marcelo Henrique de Araujo}

https://orcid.org/0000-0002-3853-9664

Doutor em Administração pela Universidade de São Paulo (USP); Pós-doutorando na Escola de Administração de Empresas São Paulo da Fundação Getulio Vargas (FGV EAESP); Pesquisador do Centro de Estudos em Microfinanças e Inclusão Financeira da Fundação Getulio Vargas (GVCemif).

E-mail: marcelo.haraujo@gmail.com

\section{Eduardo H. Diniz}

https://orcid.org/0000-0002-7950-9146

Doutor em Administração de Empresas e Professor na Escola de Administração de Empresas de São Paulo da Fundação Getulio Vargas (FGV EAESP); Pesquisador do Centro de Estudos em Microfinanças e Inclusão Financeira da FGV (GVCemif). E-mail: eduardo.diniz@fgv.br 